Ann. Zootech., 1987, 36 (1), 85-90

Note

\title{
Influence de la teneur en lignines des aliments sur la composition des digesta et la production de caecotrophes chez le lapereau
}

\author{
T. GIDENNE \\ INRA, Laboratoire de Recherches sur l'Elevage du Lapin, \\ Centre de Recherches de.Toulouse, B.P. 27, F 31326 Castanet-Tolosan Cedex
}

\begin{abstract}
Résumé
Trois lots de 36 lapereaux âgés de 6 semaines ont reçu pendant 3 semaines des rations riches en fibres, qui différaient essentiellement par leur teneur en lignines Van Soest : 7,$4 ; 12,8$ et 16 p. 100. Les contenus de l'estomac, de l'iléon, du caecum et du rectum ont été prélevés par abattages en 9 séries successives, réparties toutes les trois heures, durant 24 heures.

L'accroissement de la teneur en lignines des aliments augmente la teneur en matière sèche (MS) et réduit le taux azoté de cette dernière dans les contenus de l'estomac et du caecum. La production journalière moyenne de caecotrophes, estimée sur des lapins ne portant pas de collier, passe de 31,5 à $36,1 \mathrm{~g}$ de MS quand la teneur en lignine s'élève de 7,4 à $16,0 \mathrm{p}$. 100 . Mais quelle que soit la ration, la quantité de caecotrophes excrétée équivaut à $20 \mathrm{p}$. 100 de l'intégré sec total (aliment et caecotrophes), et 29 p. 100 de l'ingéré azoté total. Dans l'estomac, les caecotrophes représentent, à 12 heures, $70 \mathrm{p} .100$ du contenu sec, et seulement $25 \mathrm{p} .100$ à 18 heures.
\end{abstract}

Mots clés : Lapin, lignines, caecotrophie, contenus digestifs.

\section{Introduction}

Chez le lapin, la composition des contenus digestifs varie au cours du nycthémère en relation avec la pratique de la caecotrophie (CATAla, 1976). Or, par des abattages d'animaux répartis à différents moments de la journée, outre la composition des contenus digestifs, il est possible de connaître la quantité de caecotrophes émise chez des lapins libres de pratiquer la caecotrophie (non porteurs de colliers). Il suffit pour cela que ces animaux aient reçu, un aliment contenant un marqueur du transit (GIDENNE \& PonCET, 1985). Nous avons'appliqué cette technique pour étudier l'influence de l'accroissement de la teneur en lignines dans l'aliment, d'une part sur la composition des digesta (estomac, iléon, caecum, rectum), et d'autre part sur la quantité de caecotrophes produits au cours d'un cycle de 24 heures. 


\section{Matériel et méthodes}

108 lapereaux des deux sexes, de race Néozélandaise blanche, ont été répartis en trois lots de 36. Ils ont été élevés en cages individuelles à partir de l'âge de 6 semaines et ont reçu à volonté, pendant trois semaines, un des trois aliments à base de luzerne déshydratée et/ou de pellicules de colza.

Ces trois aliments riches en constituants pariétaux étaient isoazotés $(14,5$ p. 100 de protéines brutes), isocellulosiques (26,0 p. 100 de cellulose Van Soest) et ils différaient essentiellement par leur taux de lignines Van Soest : 7,4 (lot B); 12,8 (lot M) ; $16,0$ p. 100 (lot $\mathrm{H})$. Les trois aliments contenaient un marqueur de transit : le chrome mordancé sur le foin de luzerne.

A l'âge de 9 semaines, les animaux ont été abattus, en 9 séries successives réparties toutes les 3 heures de $9 \mathrm{~h}$ à $9 \mathrm{~h}$ le lendemain. Immédiatement après sacrifice, le tube digestif complet a été isolé. L'estomac, les 60 derniers centimètres de l'iléon, le caecum et les 30 derniers centimètres du tube digestif ont été pesés pleins, puis la totalité de leur contenu a été prélevée.

A chaque heure d'abattage, les contenus digestifs (digesta) ont été pesés individuellement puis regroupés pour chaque lot, par site digestif.

Les teneurs en cellulose et lignines de l'aliment ont été mesurées selon la méthode de Van Soest \& WINE (1967).

Les estimations du contenu stomacal en caecotrophes, en fonction de l'heure, ainsi que l'estimation de la production moyenne journalière en caecotrophes ont été effectuées selon la méthode décrite par Gidenne \& Poncet (1985). Cette estimation est réalisée sur des animaux libres d'ingérer leurs caecotrophes. Par contre, elle nécessite les mesures suivantes, lors de l'abattage :

- mesure du contenu total sec de l'estomac;

- mesure de la concentration moyenne en marqueur de l'estomac, des caecotrophes et de l'aliment.

Le taux de récupération du marqueur (chrome mordancé) est calculé après deux récoltes totales de fèces de 4 jours (5 lapereaux/lot).

\section{Résultats - discussion}

Le taux de récupération du marqueur est, en moyenne, sur les trois lots de $98,8 \pm 4,5$ p. 100 . Le marqueur est donc fiable et nous pouvons alors estimer correctement la production journalière moyenne de caecotrophes : QC. Elle passe de 31,5 à $36,1 \mathrm{~g} \mathrm{MS} / \mathrm{j}$ quand la teneur en lignines de l'aliment passe de 7,4 à 16 p. 100 (tabl. 1). QC équivaut à 20,5 p. 100 de l'ingéré total sec (aliment plus caecotrophes), quelle que soit la ration. De plus, QC équivaut à 29,0 p. 100 de l'ingéré azoté total. Cette estimation souligne à nouveau l'intérêt de la caecotrophie au plan de la nutrition azotée du lapin (Thacker \& BrandT, 1955 ; ... ; Jecsai et al., 1985). Nous n'observons pas une modification de cet apport azoté par les caecotrophes en fonction de la teneur en 


\section{TABleau 1}

Effet de la teneur en lignines de la ration sur la production journalière de caecotrophes (QC) et le contenu de l'estomac.

Daily caecotrophe production (QC) and stomachal content according to the lignin content of the diet.

\begin{tabular}{|c|c|c|c|c|}
\hline & Lot $B$ & Lot $\mathbf{M}$ & Lot $\mathrm{H}$ & $\begin{array}{l}\text { Effet } \\
\text { régime } \\
\text { Effect of } \\
\text { the diet }\end{array}$ \\
\hline $\begin{array}{l}\text { Quantité d'aliment ingérée }(\mathrm{g} \mathrm{MS} / \mathrm{j}) \\
\text { Voluntary feed intake }(g \mathrm{DM} / \mathrm{d})\end{array}$ & $125^{\mathrm{a}}$ & $129^{\mathrm{b}}$ & $136^{\mathrm{b}}$ & $* *$ \\
\hline $\mathrm{OC}(\mathrm{g} M S / \mathrm{j})-(\mathrm{g} D M / d)$ & 31,5 & 33,1 & 36,1 & $\mathrm{NC}$ \\
\hline $\begin{array}{l}\text { Contenu stomacal moyen en aliment } \\
\text { (g MS) } \ldots \ldots \ldots \ldots \\
\text { Stomachal content of food }(g \quad \dot{D M})\end{array}$ & $7,8^{\mathrm{a}}$ & $13,2^{\mathrm{b}}$ & $14,0^{\mathrm{b}}$ & $* *$ \\
\hline $\begin{array}{l}\text { Contenu stomacal moyen } \\
\text { en caecotrophes }(\%) \text { (1) . . . . . . . . } \\
\text { Stomachal content of caecotrophes }(\%)\end{array}$ & 29,1 & 29,7 & 30,8 & NS \\
\hline
\end{tabular}

(1) Valeur exprimée en g (MS) pour $100 \mathrm{~g}$ (MS) de contenu stomacal total - Value expressed as $\mathrm{g}$ (DM) for $100 \mathrm{~g}$ of total stomachal content.

$a, b$ : Les valeurs d'une même ligne ayant en indice une lettre différente diffèrent significativement au seuil $\mathbf{P}=0,05$ - Values, in a same line, followed by unlike superscripts are significantly different $(P<0.05)$.

$\mathrm{NC}$ : L'effet moyen de l'aliment n'est pas calculable car les valeurs correspondent à une estimation moyenne pour chaque lot (méthode de calcul décrite par GIDENne \& PONCET, 1986) - The values are mean estimation for each diet (method described by GIDENNE \& PONCET, 1986). Thus, statistical effect of the diet is not calculable.

lignines. Falcao E Cunha \& Lebas (1986) observent, par contre, une influence des lignines sur ce paramètre, mais cet effet est positif ou négatif selon l'origine botanique des lignines.

Quand la teneur en lignines de la ration s'élève, la teneur en MS du contenu de l'estomac et du caecum s'élève aussi, alors que leur teneur en protéines brutes (MAT) diminue (tabl. 2). Or, la teneur en AGV totaux mesurée sur les contenus de caecum (Gidenne, 1986) augmente avec le taux de lignines. Un accroissement de la dégradation de l'azote par une flore caecale plus active avec l'aliment $\mathrm{H}$ pourrait expliquer cette baisse de la teneur en MAT dans le caecum. Ceci est d'ailleurs accompagné d'une hausse (quoique non significative) de la teneur en ammoniac avec l'aliment $\mathrm{H}$ (GidenNe, 1986).

La quantité de caecotrophes présents dans l'estomac varie significativement en fonction de l'heure de prélèvement (fig. 1). A partir de deux points d'abattage, Catala (1976) observe une réplétion de l'estomac, liée à l'ingestion de caecotrophes. En accord avec cet auteur, nos résultats, obtenus sur un nycthémère complet, permettent de préciser que le contenu stomacal est maximum à $12 \mathrm{~h}$, à la fin de l'ingestion des caecotrophes. Ces derniers représentent alors 70 p. 100 en moyenne (pour les trois lots) du contenu sec total de l'estomac. Les caecotrophes sont ensuite évacués assez rapidement, car à $18 \mathrm{~h}$, ils ne représentent en moyenne que $25 \mathrm{p} .100$ du contenu gastrique. 
TABLEAU 2

Effet de la teneur en lignines de la ration sur les moyennes des teneurs en MS, MAT et $\mathrm{Cr}_{2} \mathrm{O}_{3}$ des contenus digestifs aux différents sites de prélèvement.

Mean concentrations in dry matter (DM), crude protein and $\mathrm{Cr}_{2} \mathrm{O}_{3}$ of digesta at the different collection sites according to the lignin content of the diet.

\begin{tabular}{|c|c|c|c|c|}
\hline & Estomac & Iléon & Caecum & Rectum \\
\hline 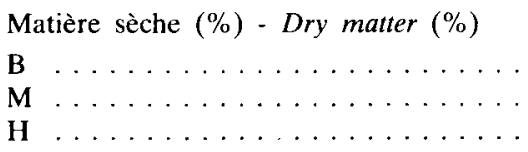 & $\begin{array}{l}13,0^{\mathrm{a}} \\
20,2^{\mathrm{b}} \\
20,9^{\mathrm{b}}\end{array}$ & $\begin{array}{l}14,3 \\
14,4 \\
15,8\end{array}$ & $\begin{array}{l}20,7^{\mathrm{a}} \\
22,6^{\mathrm{b}} \\
23,3^{\mathrm{b}}\end{array}$ & $\begin{array}{l}36,6 \\
33,5 \\
34,5\end{array}$ \\
\hline F régime ${ }^{(1)}-$ Diet & $* *$ & NS & $*$ & NS \\
\hline 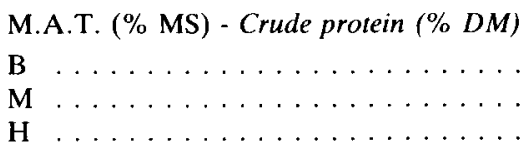 & $\begin{array}{l}21,5^{\mathrm{b}} \\
18,3^{\mathrm{a}} \\
18,2^{\mathrm{a}}\end{array}$ & $\begin{array}{l}26,3 \\
24,2 \\
22,7\end{array}$ & $\begin{array}{l}27,0^{\mathrm{h}} \\
26,3^{\mathrm{h}} \\
25,3^{\mathrm{i}}\end{array}$ & $\begin{array}{l}17,3 \\
16,2 \\
17,9\end{array}$ \\
\hline F régime ${ }^{(1)}-$ Diet & $* *$ & NS & $* *$ & NS \\
\hline
\end{tabular}

$\mathrm{a}, \mathrm{b}$ : Les valeurs d'une même colonne ayant en indice une lettre différente, diffèrent significativement au seuil $\mathbf{P}=0,05$ - Values in a column followed by unlike superscripts are significantly different $(P<0.05)$.

(1) Statistical effect of the diet.

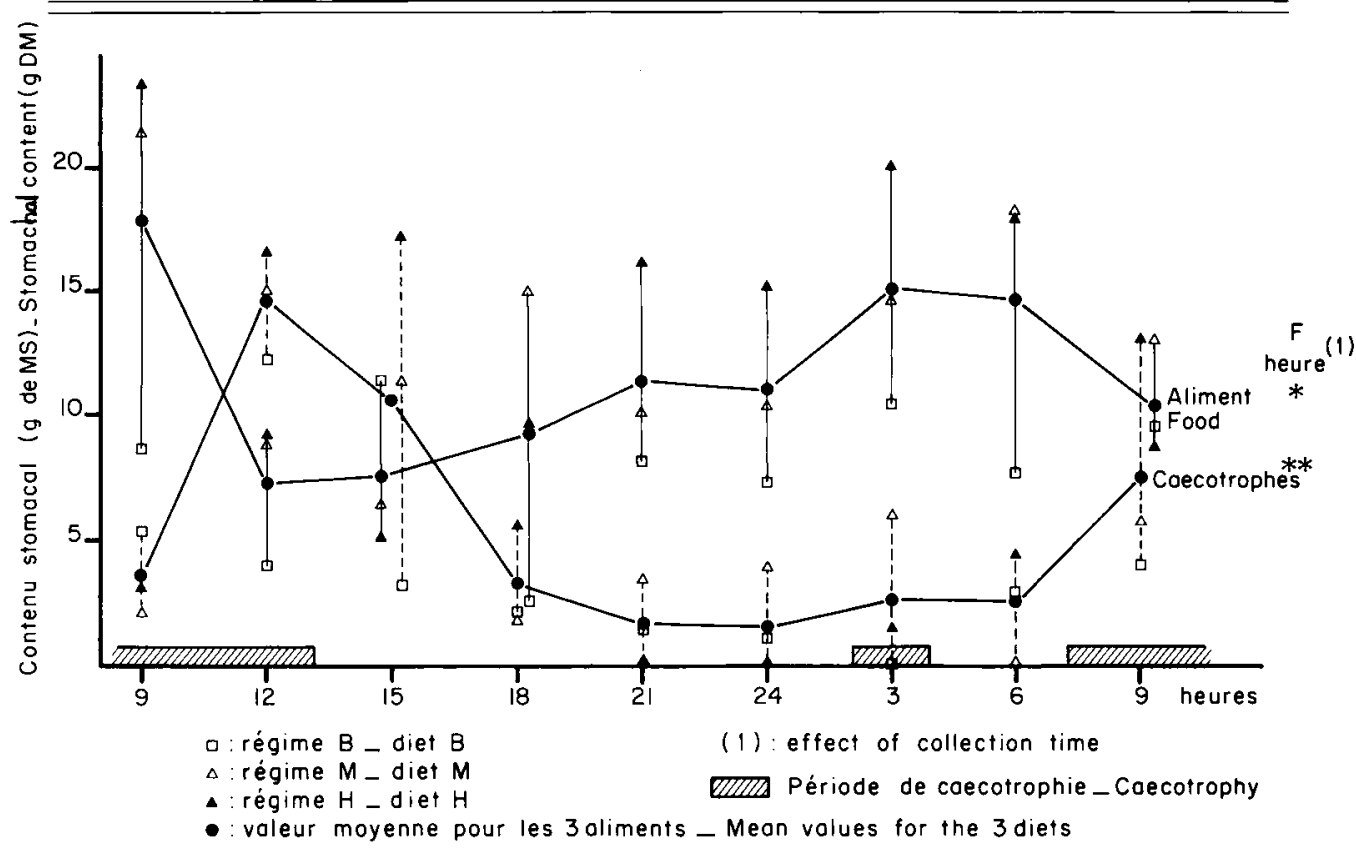

FIG. 1

Evolution nycthémérale du contenu moyen sec calculé en caecotrophes et en aliment de l'estomac, pour les 3 aliments expérimentaux.

Circadian changes in calculated dry matter content (food and caecotrophes) of the stomach, according to the diet. 
Le minimum quantitatif est observé entre $21 \mathrm{~h}$ et $24 \mathrm{~h}$. Le contenu moyen en aliment de l'estomac évolue en opposition avec le contenu en caecotrophes : minimum à $12 \mathrm{~h}$, puis hausse régulière jusqu'à $3 \mathrm{~h}$ (fig. 1).

Reçu en mars 1986.

Accepté en novembre 1986.

\section{Summary \\ Effect of dietary lignin content on digesta composition and soft faeces production in the young rabbit}

Three groups of thirty six 6-week old young rabbit were fed for three weeks fibre-rich diets with different Van Soest lignin contents : 7.4, 12.8 and 16 p. 100. Animals were slaughtered in 9 series at 3-hour intervals over a 24-h period to collect stomach, ileum, caecum and rectum contents.

The higher dietary lignin content increased dry matter (DM) content and reduced protein level of dry matter in stomach and caecum contents. The average daily production of soft faeces in rabbits prevented from caecotrophy by collars increased from 31.5 to $36.1 \mathrm{~g}$ DM when lignin content increased from 7.4 to $16.0 \mathrm{p}$. 100. However, whatever the diet, soft faeces production represented 20 p. 100 of total dry voluntary feed intake (diet and soft faeces) and 29 p. 100 of total protein intake. In the stomach, soft faeces represented at $12 \mathrm{~h}, 70 \mathrm{p} .100$ of dry content and only $25 \mathrm{p} .100$ at $18 \mathrm{~h}$.

Key words : Rabbit, lignin, caecotrophy, digesta contents.

\section{Références bibliographiques}

Catala J., 1986. Etudes sur les répartitions hydrique, pondérale et azotée dans le matériel digestif chez le lapin, en relation avec la dualité de l'élimination fécale. $1^{\text {er }}$ Congrès Int. Cunicole, Dijon, communication $\mathrm{n}^{\circ} 58$.

Falcao E Cunha L., LeBas F., 1986. Influence chez le lapin adulte de l'origine et du taux de lignine alimentaire sur la digestibilité de la ration et l'importance de la caecotrophie. $4^{\text {es }}$ Journées de la Recherche Cunicole en France, 10-11 décembre 1986, communication n" 8.

Gidenne T., Poncet C., 1985. Digestion chez le lapin en croissance d'une ration à taux élevé de constituants pariétaux : étude méthodologique pour le calcul de la digestibilité apparente, par segment digestif. Ann. Zootech., 34, 429-446.

GidenNE T., 1986. Evolution nycthémérale des produits de la fermentation bactérienne dans le tube digestif du lapin en croissance. Relations avec la teneur en lignines de la ration. Ann. Zootech., 35, 121-136.

Jecsai J., Teleki M., Juhasz B., 1985. Effect of caecotrophy on protein and amino acid metabolism of Angora rabbits. Acta Vet. Hung., 33, 51-57.

Thacker E.J., Brandt C.S., 1955. Coprography in the rabbit. J. Nutr., 55, 375-385.

VAN Soest P.J., WINE R.H., 1967. Use of detergents in the analysis of fibrous feeds. IV. Determination of plant celle-wall constituent. J. Ass. Off. Agric. Chem., 50, 50-55. 